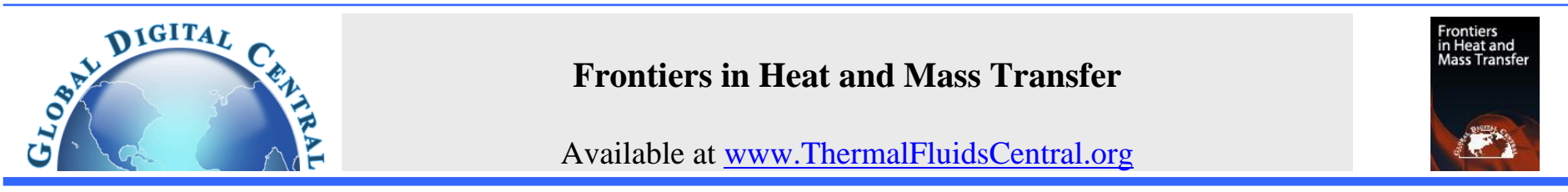

\title{
MHD FLOW OF CARREAU NANOFLUID EXPLORED USING CNT OVER A NONLINEAR STRETCHED SHEET
}

\author{
P.S.S. Nagalakshm* and N. Vijaya \\ Department of Mathematics, Koneru Lakshmaiah Education Foundation, Deemed to be University, Vaddeswaram, Guntur, Andhra
}

Pradesh-522502,India

\begin{abstract}
In the present investigation is to magnetohydrodymaics (MHD) radiative flow of an incompressible steady flow of Carreau nanofluid explored with carbon nanotubes. The boundary layer flow and heat transfer to a Carreau nanofluid model over a non- linear stretching surface is introduced. The Carreau model, adequate for many non-Newtonian fluids is used to characterize the behavior of the fluids having shear thinning properties and fluids with shear thickening properties for numerical values of the power law exponent $n$. The modeled boundary layer conservation equations are converted to non-linear coupled ordinary differential equations by a suitable transformation.R language with bvp solver was adopted to obtained numerical solutions of the resulting equations by using the Runge-Kutta method along with shooting technique. This analysis reveals many important physical aspects of flow and heat transfer. Computations are performed for different values of the stretching parameter(m),the Weissenberg number (We) and the Prandtl number (Pr).The obtained results show that the velocity of shear thinning fluid is depressed by the Weissenberg number while contrasting behavior for the shear thickening fluid is observed. A comparison with previously published data in limiting cases is performed and they are in excellent agreement.
\end{abstract}

Keywords: Shear thinning and Shear thickening,Radiation,Runge-Kutta method,R language.

\section{INTRODUCTION}

It is renowned fact that the magnetohydrodynamic (MHD), which is the science of motion of electrically conducting fluids, is one of the thrust areas of modern research. The elementary examples of electrically conducting fluids include plasmas, liquid metals (mercury or liquid sodium) and electrolytes. The basic theme of MHD is that if an electrically conducting fluid is placed in magnetic field then motion of fluid may create a force called electromotive force. The electromotive force has the ability to induce current. Ever since then, this field has a broad spectrum of science and engineering, specifically in geophysics, fusion reactors, dispersion of metals, modern metallurgy and MHD generators etc. Moreover, MHD flows are of immense concern in problems related with physiological fluids. Pavlov (1974) was the pioneer who discussed the influence of magnetic field on MHD flow past a stretching surface. Another eminent contribution was given by Andersson (1992) who examined the MHD flow of a viscous fluid. Nanofluids can be described as a colloidal suspension of nanoparticles $(1-100 \mathrm{~nm})$ scattered uniformly in a base fluid. The nanoparticles are made from materials that are chemically stable like metals, oxides ceramics, carbides and non metals such as graphite and carbon nanotubes. Nanotubes are affiliates of the fullerene structural family. Their name arises from their long, resonating edifice with the ramparts formed by one-atom-thick sheets of carbon,called graphene. These sheets are rolled at specific and discrete ("chiral") angles, and the combination of the rolling angle and radius decides the nanotube properties;for example,whether the individual nanotube shell is a metal or semiconductor. Nanotubes are categorized as single-walled nanotubes (SWNTs) and multi-walled nanotubes (MWNTs). The commonly used base fluids are ethylene, water, oils and lubricants. Choi (1995) who was working with a group of Argonne National Laboratory USA,introduced the term Nanofluid. Nanofluids enhance the thermophysical properties of the base fluids used. Nanofluids have a wide range of practical applications, they can be used as smart materials to work as heat valve to control the flow of heat. Advanced electronic gadgets experience thermal management challenges from reduction of accessible surface area for heat and high level of heat generation. These challenges can be avoided by either increasing the capacity of heat transfer or by finding an optimum geometry of cooling appliances. Due to their high thermal conductivity and increased heat transfer coefficient, nanofluids can be used for liquid coolant of computer processors. In presence of magnetic field, nanofluids have several engineering, industrial and biomedical applications such as cooling of nuclear reactors by liquid sodium and induction flow meter, magnetogravimetric separations, smart fluids for vibration damping, aerodynamic sensors magnetic drug targeting, nanocryosurgery and nanodrug delivery.

Till date, as a consequence of enormous industrial applications the notion of non-Newtonian fluids have dragged much insight when equated to Newtonian liquids. Moreover, plenty of experimental and notional studies have been executed to scrutinize the mechanism of non-Newtonian

${ }^{\dagger}$ Corresponding author. Email: sathya.krishnat@gmail.com 
transport owing to their extensive applications in numerous genetic and developed progressions, materials and motorized engineering. Numerous materials, for instance biomedical flows, splashes, bio-fluids in genetic material and polymers are identified as non-Newtonian fluids. The non-Newtonian Carreau fluid model is the combination of Newtonian and power-law models. This fluid model is capable of describing both shear thinning and shear thickening phenomena. In spite of all such concentrations, innumerable investigators are quiet engaged to scrutinize the analysis of non-Newtonian liquids under diverse prospective. Rheological equations from molecular network theories was presented by Carreau (1972). Hydromagnetic natural convection from an isothermal inclined surface adjacent to a thermally stratified porous medium has been studied by Chamkha (1997). "Unsteady flow and heat transfer on a semiinfinite flat plate with an aligned magnetic field (Takhar et al., 1999)". "Unsteady three-dimensional MHD-boundary-layer flow due to the impulsive motion of a stretching surface (Takhar et al., 2001)". Magnetohydrodynamic three dimensional flow and heat transfer over a stretching surface in a viscoelastic fluid was explored by Ahmad and Nazar (2011). Boundary layer flow and heat transfer to Carreau fluid over a nonlinear stretching sheet was presented by Khan and Hashim (2015). Numerical simulation of water based magnetite nanoparticles between two parallel disks were simulated by Haq et al. (2016). Impact of nonlinear thermal radiation and gyrotacticmicroorganisms on the MagnetoBurgers nanofluid were studied by Khan et al. (2017). A microscopic study of MHD fractional inertial flow through Forchheimer medium were shown by Anwar and Rasheed (2017). Carbon nanotubes significance in Darcy-Forchheimer flow were established by Hayat et al. (2018). Lu et al. (2018) investigated the effect of a numerical treatment of MHD radiative flow of Micropolar nanofluid with homogeneous-heterogeneous reactions past a nonlinear stretched surface. "Unsteady squeezing carbon nanotubes based nano-liquid flow with Cattaneo-Christov heat flux and homogeneous-heterogeneous reactions (Lu et al., 2019)". Numerical study of Carreau nanofluid flow past vertical plate with the Cattaneo-Christov heat flux model was shown by Vasu and Ray (2019).

The objective of present investigation is to discuss the boundary layer flow and heat transfer of Carreau nanofluid model over a non-linear stretching surface. We obtained the boundary layer equations for the velocity field of the Carreau nanofluid explored with CNT. This model which, in comparison with the Newtonian case involves three additional dimensionless parameters, allows the characterization of a wide variety of shear-thinning fluids. The numerical method namely Runge-Kutta shooting method is used to solve the non-linear governing problem. The obtained results for both the fluid velocity and its temperature are presented for different values of parameters entering in the problem. The effects of engineering parameters, like the Weissenberg number (We), the power law index $\mathrm{n}$ and the Prandtl number (Pr) on the local skin friction and local Nusselt number are presented numerically in tabular form.

\section{MATHEMATICAL FORMULATION}

Consider the problem of an incompressible two dimensional flow of a generalized non Newtonian fluid, namely Carreaue nanofluid, due to a stretching sheet which coincides with the plane $y=0$. It is assumed that the flow being confined to $y>0$. A uniform magnetic field $B_{0}$ is applied parallel to the $y$-axis . The induced magnetic field is neglected for small magnetic reynold number. Heat and mass transfer characteristics are taken into account in the presence of thermal radiation and thermophoresis effects. The uniform temperature of the surface $\mathrm{T}_{w}$ is larger than ambient fluid temperature $\mathrm{T}_{\infty}$ and it is moving with nonlinear velocity $\mathrm{U}_{w}$. The species concentration at the surface $\mathrm{C}_{w}$ and ambient concentration $\mathrm{C}_{\infty}$ are constants. The following governing equations are introduced (Khan and Hashim, 2015)

$$
\frac{\partial p}{\partial x}+\frac{\partial q}{\partial y}=0
$$

$$
\begin{aligned}
p \frac{\partial p}{\partial x}+q \frac{\partial p}{\partial y} & =\nu_{n f} \frac{\partial^{2} p}{\partial x^{2}}\left(1+\tau^{2}\left(\frac{\partial p}{\partial y}\right)^{2}\right)^{\left(\frac{n-1}{2}\right)} \\
& +\nu_{n f}(n-1) \tau^{2}\left(\frac{\partial p}{\partial y}\right)^{2} \frac{\partial^{2} p}{\partial x^{2}}\left(1+\tau^{2}\left(\frac{\partial p}{\partial y}\right)^{2}\right)^{\left(\frac{n-3}{2}\right)} \\
& -\frac{\sigma_{n f}}{\rho_{n f}} B^{2} p+g\left(\beta_{T}\left(T-T_{\infty}\right)+\beta_{C}\left(C-C_{\infty}\right)\right)
\end{aligned}
$$

$$
\begin{aligned}
\left(p \frac{\partial T}{\partial x}+q \frac{\partial T}{\partial y}\right)\left(\rho c_{p}\right)_{n f} & =k_{n f} \frac{\partial^{2} T}{\partial y^{2}}+\mu_{n f}\left(\frac{\partial p}{\partial y}\right)^{2}-\frac{\sigma_{n f}}{\rho_{n f}} B^{2} p^{2} \\
& +\frac{16 \sigma^{s} T_{\infty}^{3}}{3 k^{s}} \frac{\partial^{2} T}{\partial y^{2}}
\end{aligned}
$$$$
p \frac{\partial C}{\partial x}+q \frac{\partial C}{\partial y}=D \frac{\partial^{2} C}{\partial y^{2}}-\frac{\partial}{\partial y}\left(V_{T}\left(C-C_{\infty}\right)\right)
$$

where $\mathrm{p}$ and $\mathrm{q}$ are the velocity components in $\mathrm{x}$ and $\mathrm{y}$ directions respectively, $\nu_{n f}$ kinematic nanofluid viscosity, $\tau$ material time constant, $\mathrm{n}$ expression power law index , $\rho_{n f}$ density of nanofluid, $\mathrm{k}_{n f}$ thermal conductivity of nanofluid, $\sigma_{n f}$ electrical conductivity of nanofluid , $g$ the gravitational acceleration $\beta_{T}$ and $\beta_{C}$ thermal expansion coefficients of temperature and concentration, $\mathrm{c}_{p}$ specific heat, $\sigma^{s}$ the StefanBoltzmann constant, $\mathrm{k}^{s}$ the mean absorption coefficient, $\mathrm{D}$ the diffusion coefficient and $B$ nonlinear uniform magnetic field $V_{T}$ the thermophoretic velocity defined as

$$
B=B_{0} x^{\left(\frac{m-1}{2}\right)}, \quad V_{T}=-k_{1} \frac{\nu}{T_{r e f}} \frac{\partial T}{\partial y}
$$

where $\mathrm{k}_{1}$ is the thermophoretic coefficient, $\mathrm{T}_{r e f}$ is the reference temperature.

Corresponding boundary conditions are

$$
\begin{aligned}
& p=p_{w}(x)=b x^{m}, q=0, T=T_{w}, C=C_{w} \quad \text { at } y=0 \\
& p \rightarrow 0, T \rightarrow T_{\infty}, C \rightarrow C_{\infty} \quad \text { as } \quad y \rightarrow \infty
\end{aligned}
$$

where $\mathrm{b}$ and $\mathrm{m}$ are positive real numbers relating to the stretching sheet.

Nanofluid parameters are defined as

$$
\begin{aligned}
\nu_{n f} & =\frac{\mu_{n f}}{\rho_{n f}}, \\
\rho_{n f} & =(1-\phi) \rho_{b f}+\phi \rho_{C N T}, \\
\mu_{n f} & =\frac{\mu_{b f}}{(1-\phi)^{2.5}}, \\
\left(\rho c_{p}\right)_{n f} & =(1-\phi)\left(\rho c_{p}\right)_{b f}+\phi\left(\rho c_{p}\right)_{C N T}, \\
\frac{k_{n f}}{k_{b f}} & =\frac{\left(k_{C N T}+2 k_{b f}\right)-2 \phi\left(k_{b f}-k_{C N T}\right)}{\left(k_{C N T}+2 k_{b f}\right)+\phi\left(k_{b f}-k_{C N T}\right)}, \\
\frac{\sigma_{n f}}{\sigma_{b f}} & =1+\frac{3\left(\sigma_{C N T}-\sigma_{f}\right) \phi}{\left(\sigma_{C N T}+2 \sigma_{b f}\right)-\left(\sigma_{C N T}-\sigma_{b f}\right) \phi}
\end{aligned}
$$

Now introducing the following dimensionless variables for stream function, temperature, concentration and the independent variable as:

$$
\begin{aligned}
& \psi=\sqrt{\frac{2 b \nu_{b f}}{m+1}} x^{\left(\frac{m-1}{2}\right)} f(\eta), \quad \eta=y x^{\left(\frac{m-1}{2}\right)} \sqrt{\frac{b(m+1)}{2 \nu_{b f}}} \\
& p=\frac{\partial \psi}{\partial y}, \quad q=-\frac{\partial \psi}{\partial x} \\
& \theta(\eta)=\frac{\left(T-T_{\infty}\right)}{\left(T_{w}-T_{\infty}\right)}, \quad h(\eta)=\frac{\left(C-C_{\infty}\right)}{\left(C_{w}-C_{\infty}\right)}
\end{aligned}
$$


Using the above variables into Eqs. (1)-(4) and boundary conditions (6) , governing equations are reduced as ordinary differential equations as follows

$$
\begin{gathered}
\frac{P_{1}}{P_{2}}\left(1+n W e_{x}^{2}\left(f^{\prime \prime}\right)^{2}\right)\left(1+W e_{x}^{2}\left(f^{\prime \prime}\right)^{2}\right)^{\frac{n-3}{2}} f^{\prime \prime \prime} \\
-\frac{P_{3}}{P_{1}} l_{1} M f^{\prime}+\gamma l_{1}(\theta+N h)-\beta\left(f^{\prime 2}\right)+f f^{\prime \prime}=0 \\
\left(1+\frac{4}{3 P_{5}} R\right) \theta^{\prime \prime}+\frac{P_{4}}{P_{5}}\left(\operatorname{Pr} \theta^{\prime} f^{\prime}\right) \\
+\frac{P_{1}}{P_{5}} \operatorname{Pr} E c\left(f^{\prime \prime}\right)^{2}-\frac{P_{3}}{P_{5}} M l_{1} E c \operatorname{Pr}\left(f^{\prime}\right)^{2}=0 \\
h^{\prime \prime}+S c\left(f h^{\prime}\right)-\tau^{s} S c\left(h \theta^{\prime \prime}+\theta^{\prime} h^{\prime}\right)=0
\end{gathered}
$$

and the corresponding boundary conditions

$$
\begin{aligned}
& f(0)=0 \quad, \quad f^{\prime}(0)=1 \quad, \quad \theta(0)=1 \quad, \quad h(0)=1 \quad \text { at } \quad \eta=0 \\
& f^{\prime}(\eta)=0 \quad, \quad \theta(\eta)=0 \quad, \quad h(\eta)=0 \quad \text { as } \quad \eta \rightarrow \infty
\end{aligned}
$$

where prime denotes differentiation with respect to $\eta$,

$$
\begin{aligned}
& \beta=\frac{2 m}{m+1}, \quad l_{1}=\frac{2}{m+1}, \quad W e_{x}=\left(\frac{(m+1) \tau^{2} x^{3 m-1}}{2 \nu_{f}} b^{3}\right)^{0.5}, \\
& \gamma=\frac{G r_{x}}{R e_{x}^{2}}, \quad G r_{x}=\frac{\beta_{T}\left(T_{w}-T_{\infty}\right) x^{3}}{\nu_{f}^{2}}, \quad R e_{x}=\frac{u_{w} x}{\nu_{f}}, \\
& \operatorname{Pr}=\frac{\mu c_{p}}{k}, \quad E c=\frac{u_{w}^{2}}{\left(c_{p}\right)_{f}\left(T_{w}-T_{\infty}\right)}, \quad S c=\frac{\nu_{f}}{D}, \\
& M=\frac{\sigma_{f} B_{0}^{2}}{b \rho_{f}}, \quad N=\frac{\beta_{C}\left(C_{w}-C_{\infty}\right)}{\beta_{T}\left(T_{w}-T_{\infty}\right)}, \\
& R=\frac{4 \sigma^{s} T_{\infty}^{3}}{k^{s} k}, \quad \tau^{s}=-\frac{k_{1}\left(T_{w}-T_{\infty}\right)}{T_{r e f}}
\end{aligned}
$$

Some nanofluid parameters are

$$
\begin{aligned}
& P_{1}=\frac{1}{(1-\phi)^{2.5}}, \quad P_{2}=\left[(1-\phi)+\phi \frac{\rho_{C N T}}{\rho_{b f}}\right], \\
& P_{3}=1+\frac{3\left(\sigma_{C N T}-\sigma_{b f}\right) \phi}{\left(\sigma_{C N T}+2 \sigma_{b f}\right)-\left(\sigma_{C N T}-\sigma_{b f}\right) \phi} \\
& P_{4}=\left[(1-\phi)+\phi \frac{\left(\rho c_{p}\right)_{C N T}}{\left(\rho c_{p}\right)_{b f}}\right], \\
& P_{5}=\frac{(1-\phi)+2 \phi\left(\frac{k_{C N T}}{k_{C N T}-k_{b f}}\right) \ln \left(\frac{k_{C N T}+k_{b f}}{2 k_{b f}}\right)}{(1-\phi)+2 \phi\left(\frac{k_{b f}}{k_{C N T}-k_{b f}}\right) \ln \left(\frac{k_{C N T}+k_{b f}}{2 k_{b f}}\right)}
\end{aligned}
$$

Engineering parameters likeskin friction coefficient $\mathrm{Cf}_{x}$, local Nussult $\mathrm{Nu}_{x}$ and Sherwood $\mathrm{Sh}_{x}$ numbers are defined as

$$
C f_{x}=\frac{\tau_{w}}{\rho_{f} p_{w}^{2}}, N U_{x}=\frac{x q_{w}}{k_{f}\left(T_{w}-T_{\infty}\right)}, S h_{x}=\frac{x j_{w}}{D\left(C_{w}-C_{\infty}\right)}
$$

in which $\tau_{w}, \mathrm{q}_{w}$, and $\mathrm{j}_{w}$ denote the surface shear stress, the surface heat flux and the surface mass flux respectively. Here

$$
\begin{aligned}
& \tau_{w}=\mu_{n f}\left(\frac{\partial p}{\partial y}\left(1+\tau^{2}\left(\frac{\partial p}{\partial y}\right)^{2}\right)^{\left(\frac{n-1}{2}\right)}\right)_{y=0} \\
& q_{w}=-k_{n f}\left(\frac{\partial T}{\partial y}\right)_{y=0} \quad, \quad j_{w}=-D\left(\frac{\partial C}{\partial y}\right)_{y=0}
\end{aligned}
$$

In dimensionless coordinates one can express that

$$
\begin{aligned}
& R e_{x}^{0.5} C f_{x}=\left(\frac{m+1}{2}\right)^{0.5}\left(1+w e_{x}^{2}\left(f^{\prime \prime}\right)^{2}\right)^{\frac{n-1}{2}}(1-\phi)^{-2.5} f^{\prime \prime}(0), \\
& R e_{x}^{-0.5} N u_{x}=-\left(\frac{m+1}{2}\right)^{0.5} P_{5} \theta^{\prime}(0), \\
& R e_{x}^{-0.5} S h_{x}=-\left(\frac{m+1}{2}\right)^{0.5} h^{\prime}(0)
\end{aligned}
$$

\section{NUMERICAL METHOD FOR SOLUTION}

The nonlinear differential Eqs. (9) to (11) with boundary conditions (12) have been solved numerically using Runge-Kutta fourth order method along with shooting technique using bvp solver package in $\mathrm{R}$ software. Thus, Eqs. (9) to (11) have been converted into first-order equations as follows:

$$
\begin{aligned}
& g_{1}^{\prime}=g_{2} \\
& g_{2}^{\prime}=g_{3} \\
& g_{3}^{\prime}=\frac{\beta\left(g_{2}\right)^{2}-g_{1} g_{3}+\frac{P_{3}}{P_{2}} l_{1} M g_{2}-l_{1} \gamma\left(g_{4}+N g_{5}\right)}{\frac{P_{1}}{P_{2}}\left(1+n w e_{x}^{2}\left(g_{3}\right)^{2}\right)\left(1+w e_{x}^{2}\left(g_{3}\right)^{2}\right)^{\frac{n-3}{2}}} \\
& g_{4}^{\prime}=g_{5} \\
& g_{5}^{\prime}=\frac{-\frac{P_{4}}{P_{5}}\left(\operatorname{Pr} g_{5} f_{1}\right)-\frac{P_{1}}{P_{5}} \operatorname{Pr} E c\left(g_{3}\right)^{2}+\frac{P_{3}}{P_{5}} M l_{1} \operatorname{EcPr}\left(g_{2}\right)^{2}}{\left(1+\frac{4}{3 P_{5}} R\right)} \\
& g_{6}^{\prime}=g_{7} \\
& g_{7}^{\prime}=-S c\left(g_{1} g_{7}\right)+\tau^{s} S c\left(g_{6} g_{5}^{\prime}+g_{7} g_{5}\right) \\
& \text { where } g_{1}=f, g_{2}=f^{\prime}, g_{3}=f^{\prime \prime}, g_{4}=\theta, g_{5}=\theta^{\prime}, g_{6}=h, g_{7}=h^{\prime} .
\end{aligned}
$$

Boundary conditions (12) becomes,

$$
\begin{aligned}
& g_{1}(0)=0, g_{2}(0)=1, g_{4}(0)=1, g_{6}(0)=1 \\
& g_{2}(\infty)=0, g_{4}(\infty)=0, g_{6}(\infty)=0
\end{aligned}
$$

In view of the above boundary conditions, out of seven only four initial conditions are known and solution procedure is begun by three initial guesses of $g_{3}(0), g_{5}(0), g_{7}(0)$ with the shooting method iteration to assure the iteration convergence required to satisfy asymptotically boundary conditions at infinity. Thus, in the present study the value of $\eta=\eta_{\max }$ is taken to be 10 and step-size is taken to be $\Delta \eta=0.01$ with relative error tolerance $10^{-5}$.

\section{RESULTS AND DISCUSSION}

The behavior of different variables for the velocity, thermal field, nanoparticles concentration, skin friction coefficient and local Nusselt and Sherwood numbers are investigated through plots.

\subsection{Accuracy assessment}

Accuracy of the present results are compared with results mentioned in Khan and Hashim (2015). In the absence of nanoparticles, Magnetic parameters, Weissenberg number, local buoyancy parameter, Eckert number, Schmidt number, radiation parameter, these comparisons are found to be in excellent agreement as shown in tables 1 and 2 .

\subsection{Dimensionless velocity}

Fig. 1 demonstrates that by enhancing Weissenberg number, velocity profile decreases in case of shear thinning $(n<1)$ fluid while it shows increasing behavior for shear thickening $(n>1)$ fluid. Since Weissenberg number is the ratio of the relaxation time of the fluid and a specific process time, it enhances the thickness of fluid which in-turn causes the reduction of 
velocity. Fig. 2 is plotted in order to examine the influence of magnetic field on Carreau nanofluid velocity for shear thickening fluid. It is clear from figures that the Carreau nanofluid velocity is a decreasing function of applied magnetic field. The inciting values of magnetic field parameter reflect higher values of Lorentz force. Since this force is resistive in nature, the Carreau nanofluid particles faced resistance and hence a decline in velocity curves appeared. Fig. 2 also shows that the velocity profile is increasing function of $\gamma$ due to this boundary layer increases. Invetigators observe that all curves move to zero at the threshold value of $\eta$ is 4.7. For $\eta=0$ the velocities have the same values since there is no slip condition between the nanofluid and the surface and it is due to the boundary condition at the wall.

\subsection{Dimensionless temperature}

Fig. 3 potrayed the behavior of radiation parameter(R), Prandtl number $(\mathrm{Pr})$, and magnetic parameter(M). Thermal field and layer thickness are increasing functions of $\mathrm{R}$. As expected, heat is produced due to radiation process in the working fluid, so the temperature field enhances. Temperature for Prandtl number $(\mathrm{Pr})$ is drawn in Fig. 3 . Here both temperature and layer thickness are diminished for larger Pr. Physically higher Pr lead to lower thermal diffusivity which results in the reduction of thermal field. Fig. 3 clearly indicates that the temperature and layer thickness are increased for larger magnetic parameter M. Physically higher magnetic parameter increases heat transfer coefficient which enhances the fluid temperature. Fig. 4 delineates temperature variation via Weissenberg number (we). For larger Weissenberg number (we) both temperature and layer thickness is decreased. In fact, less heat is produced through random motion of the fluid particles due to larger Weissenberg number (we) variable and consequently the temperature decreases. Invetigators observe that thermal boundary layers move to zero at the threshold value of $\eta$ is 5.7. For $\eta=0$ the temperature remains the same due to the boundary condition at the wall.

\subsection{Dimensionless concentration}

Graphical illustration for concentration against Schmidt number $(\mathrm{Sc})$, thermophoretic parameter $\left(\tau^{s}\right)$ are captured in Fig. 5. It is revealed that concentration and related layer thickness are decayed for larger Schmidt number $(\mathrm{Sc})$. Schmidt number $(\mathrm{Sc})$ is the ratio of momentum to mass diffusivities. For enhancement of Schmidt number, the mass diffusivity diminishes which is responsible in reduction of concentration.Boundary layer of concetration decreases with increasing thermophoretic parameter shown in the figure. Outcomes of generative/destructive chemical reaction $\gamma$ on concentration field are delineated in the figure. There is an enhancement in fluid concentration in view of destructive chemical reaction variable. Investigators observe that boundary layers of concentration move to zero at the threshold value of $\eta$ is 5.7. For $\eta=0$ the concentration remains the same due to the boundary condition at the wall.

\subsection{Dimensionless Engineering parameters}

Behaviors of magnetic parameter(M), Weissenberg number(We) on skin friction coefficient, local Nusselt number, and local Sherwood number are disclosed in Table. 3 .

\subsection{Different Base fluids and Carbon nanotubes}

Figs. 6-7 shows that the momentum boundary layer of water based singlewalled carbon nanotube is more when compared to kerosene and engine oil at both shear thinning and shear thickening fluid. Figs. 8-9 depict that the thermal boundary layer of water is more when compared to remaining base fluids in both shear thinning and shear thickening cases. Figs. 10-11 represent that concentration boundary layer of water is less when compared to the remaining base fluids in shear thinning but in shear thickening the concentration of boundary layer increases. Momentum boundary layer of water based multi-walled carbon nanotube is more when compared to kerosene and engine oil at both shear thinning and shear thick- ening fluid as shown in Figs. 12-13. Figs. 14-15 depict that the thermal boundary layer of water is more as compared to remaining base fluid in both shear thinning and shear thickening cases.Figs. 16-17. represents concentration boundary layer of water is less with reference to the remaining base fluids in shear thinning but in shear thickening the concentration of boundary layer increases.

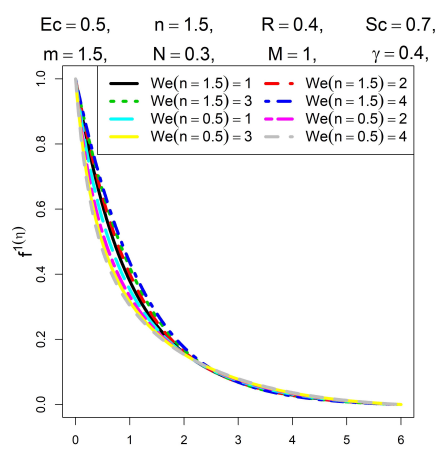

$(\eta)$

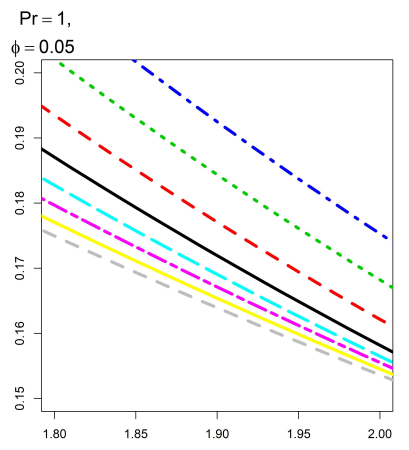

Fig. 1 Velocity profile for Shear thinning and Shear thickening fluid with Weissenberg number (We)

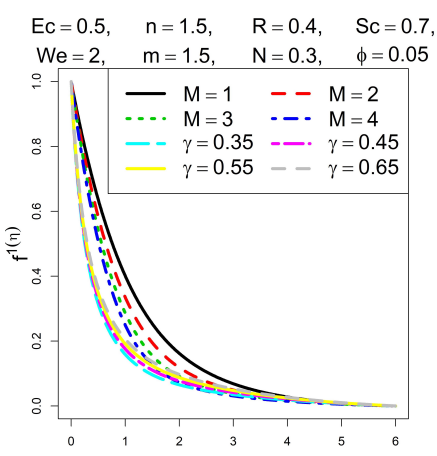

( $)$

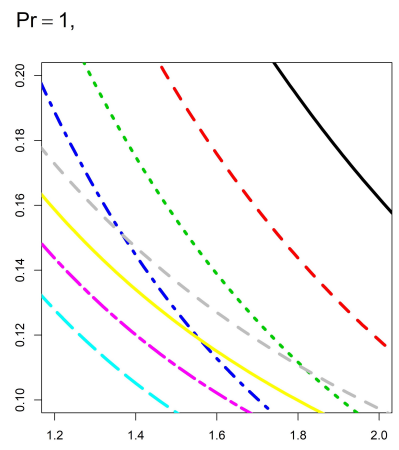

Fig. 2 Velocity profile for Shear thickening fluid with Magnetic parameter(M), and Local buoyancy parameter $(\gamma)$
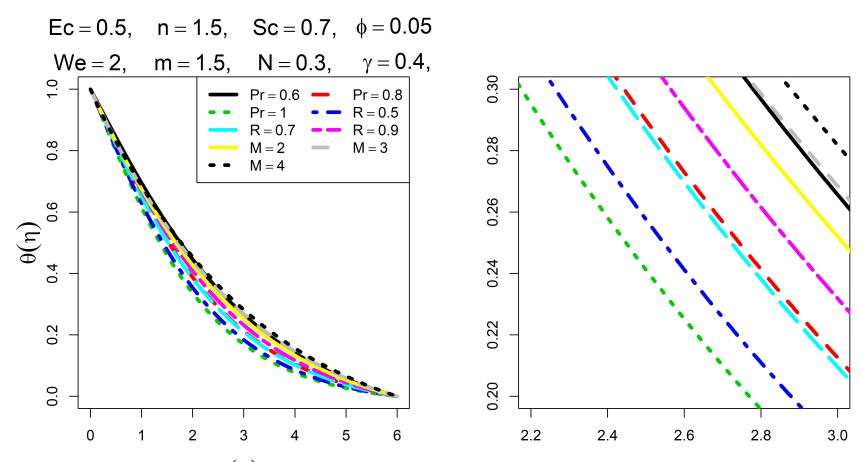

$(\eta)$

Fig. 3 Temperature profile for Shear thickening fluid with Radiation(R), Prandtl number (Pr), and magnetic parameter(M) 


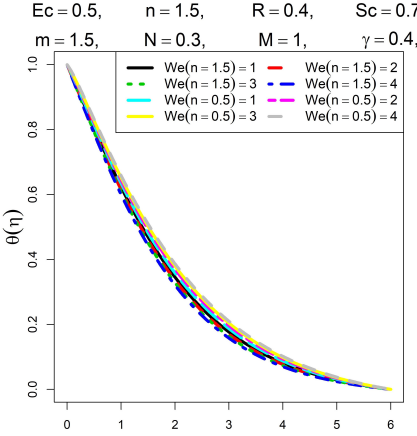

$(\eta)$

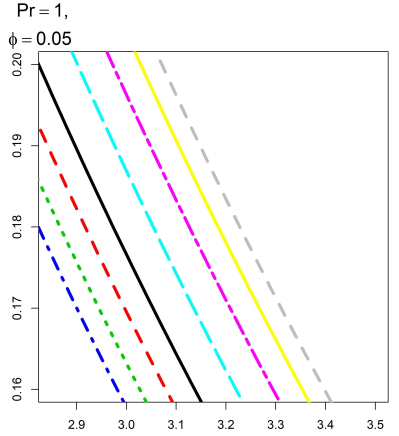

$\begin{array}{lllllll}2.9 & 3.0 & 3.1 & 3.2 & 3.3 & 3.4 & 3.5\end{array}$
Fig. 4 Temperature profile for Shear thickening and Shear thinning fluid with Weissenberg number (We)
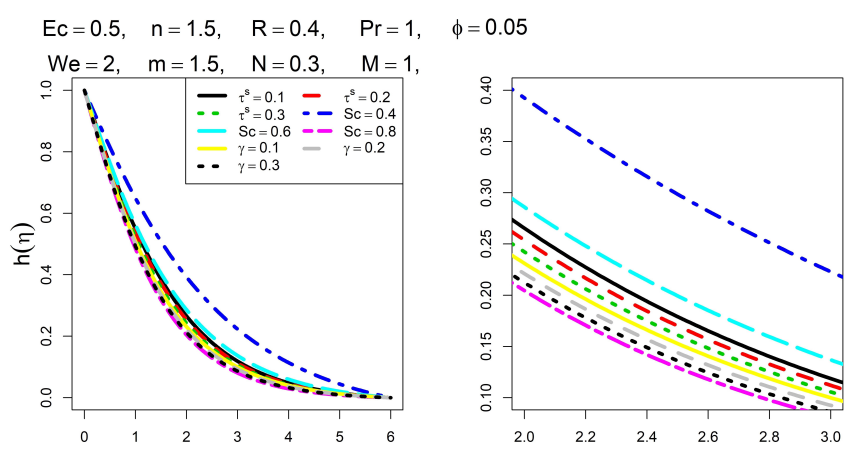

$(\eta)$

Fig. 5 Concentration profile for thermophoretic parameter $\left(\tau^{s}\right)$,Schmidt number(Sc), and Local buoyancy paramete $(\gamma)$

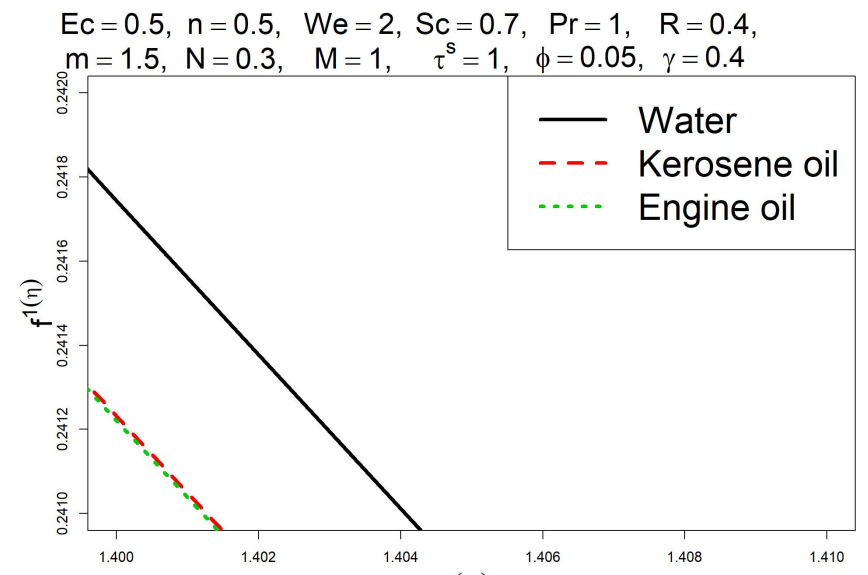

$(\eta)$

Fig. 6 Velocity profile for Shear thinning fluid with SWCNT

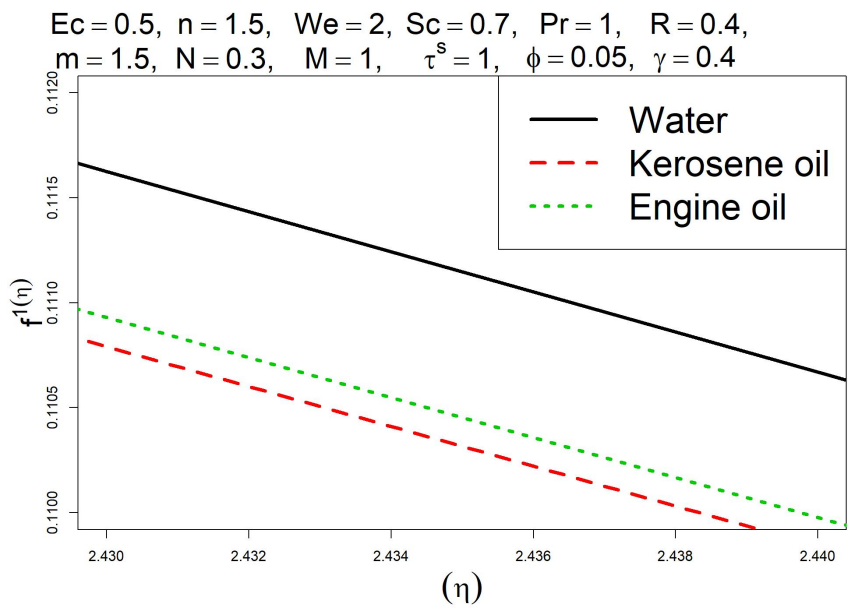

Fig. 7 Velocity profile for Shear thickening fluid with SWCNT

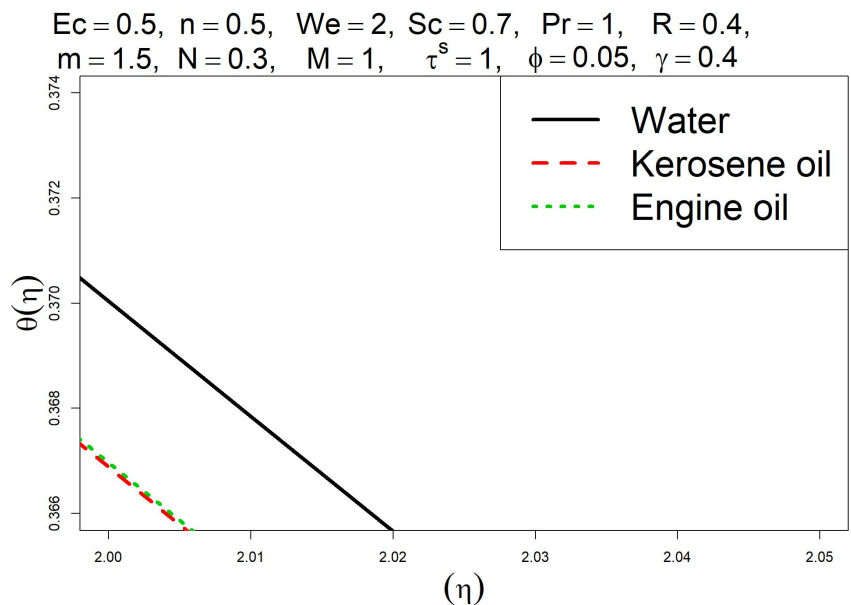

Fig. 8 Temperature profile for Shear thinning fluid with SWCNT

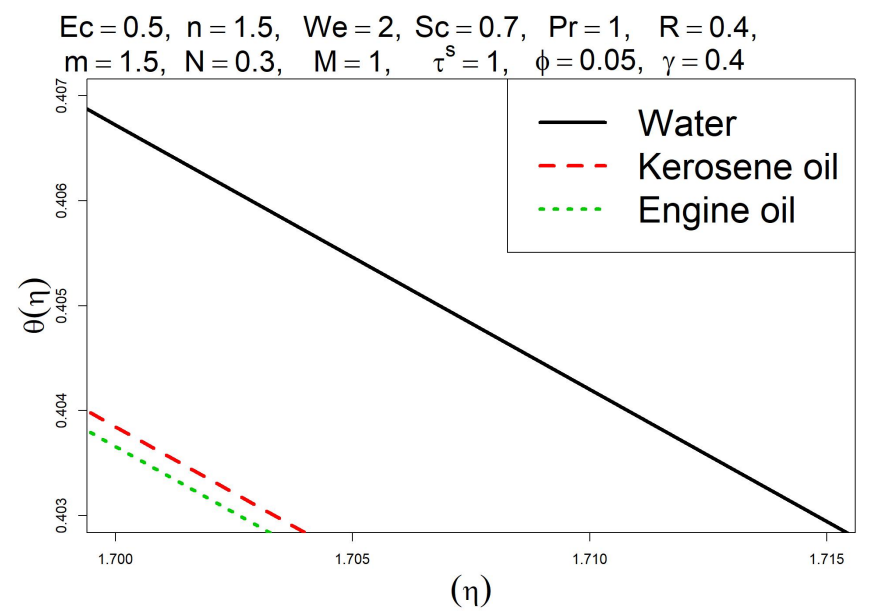

Fig. 9 Temperature profile for Shear thickening fluid with SWCNT 


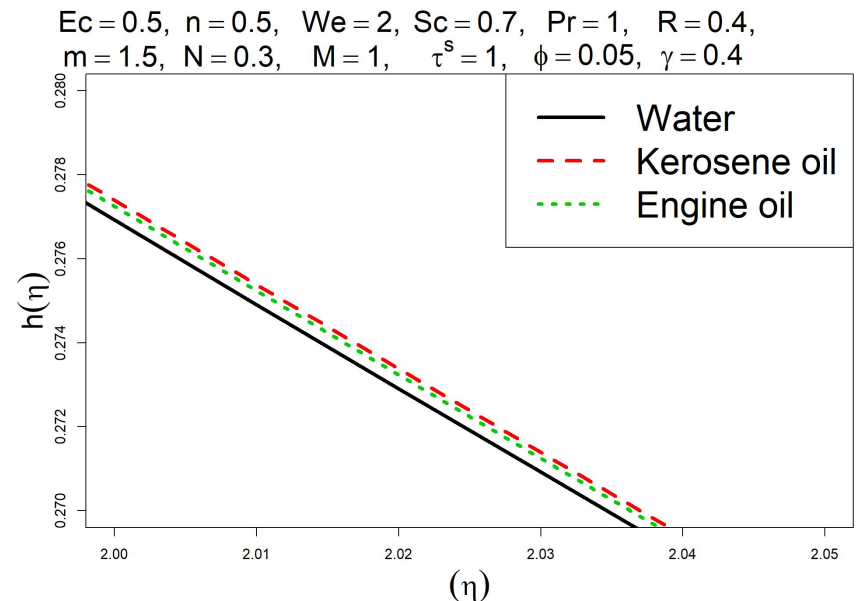

Fig. 10 Concentration profile for Shear thinning fluid with SWCNT

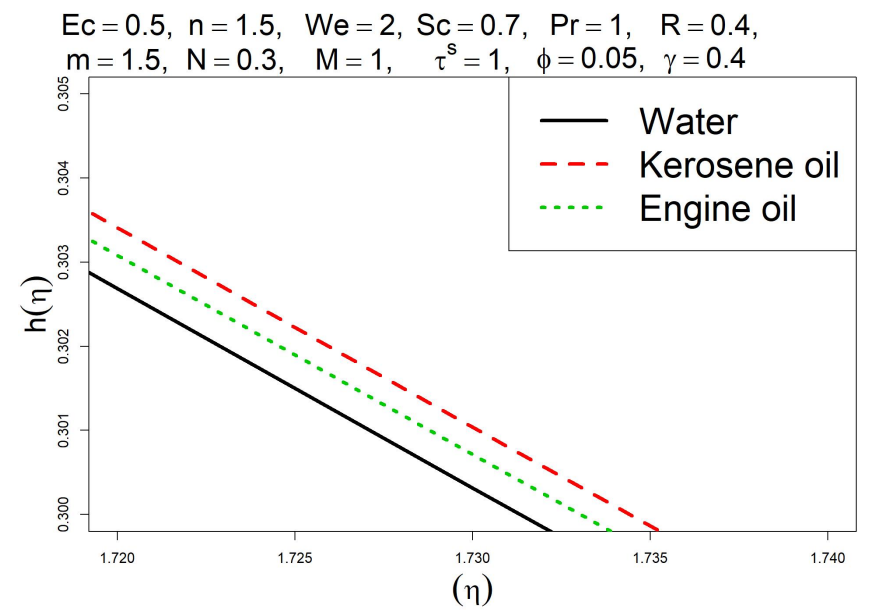

Fig. 11 Concentration profile for Shear thickening fluid with SWCNT

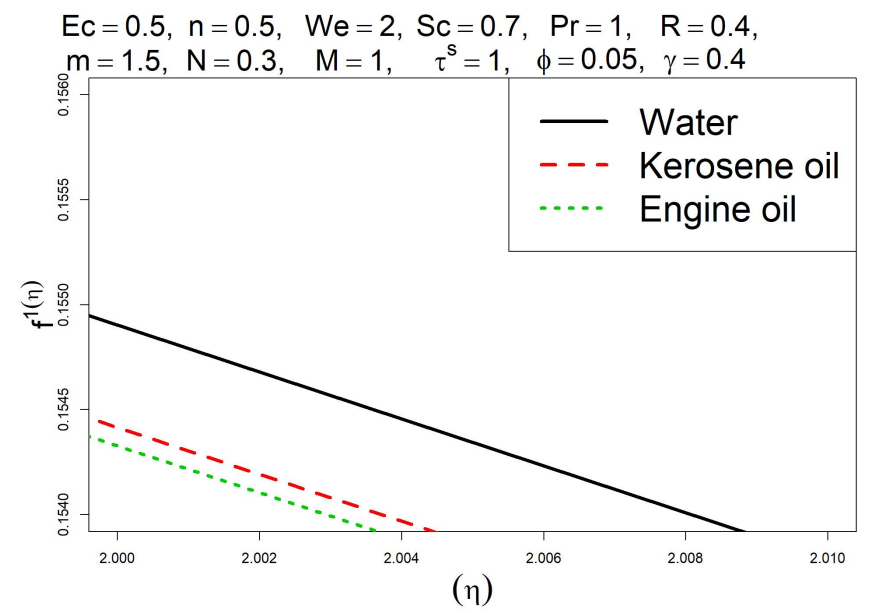

Fig. 12 Velocity profile for Shear thinning fluid with MWCNT

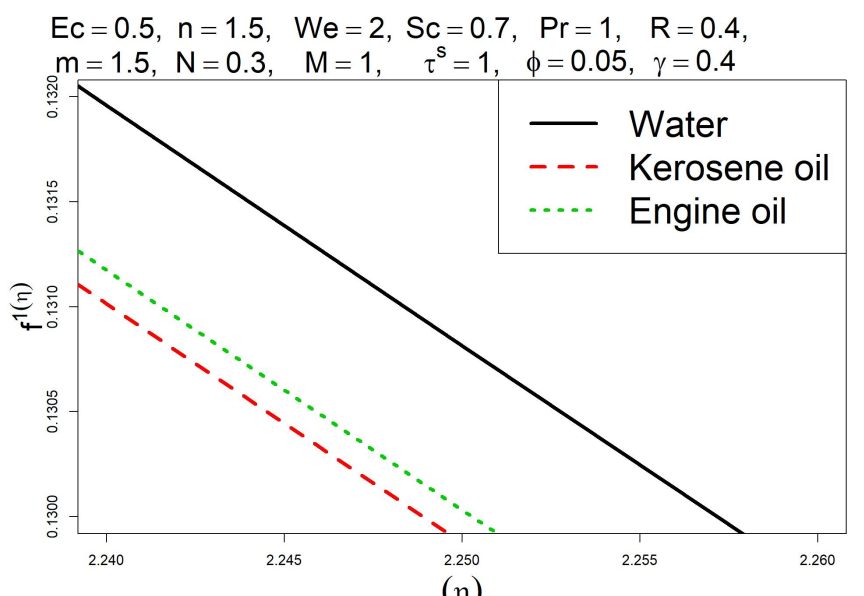

$(\eta)$

Fig. 13 Velocity profile for Shear thickening fluid with MWCNT

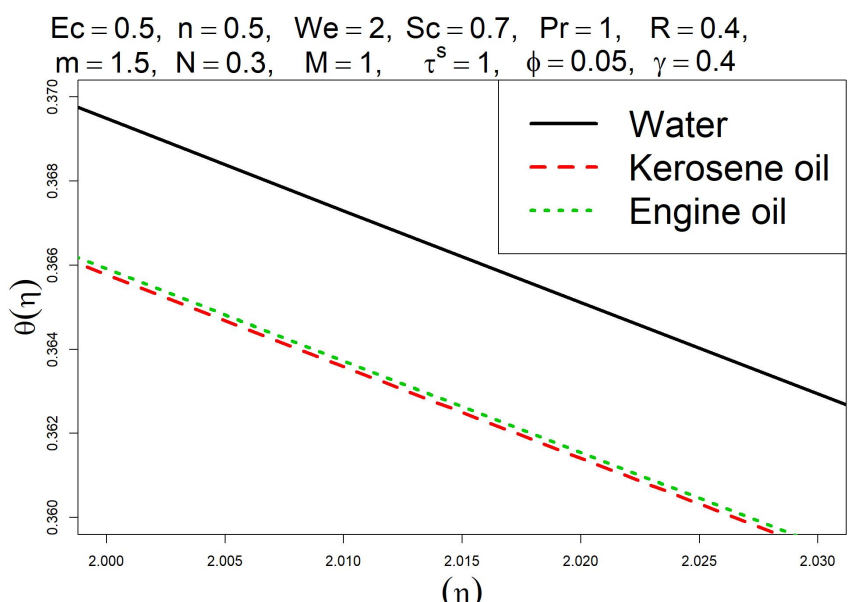

Fig. 14 Temperature profile for Shear thinning fluid with MWCNT

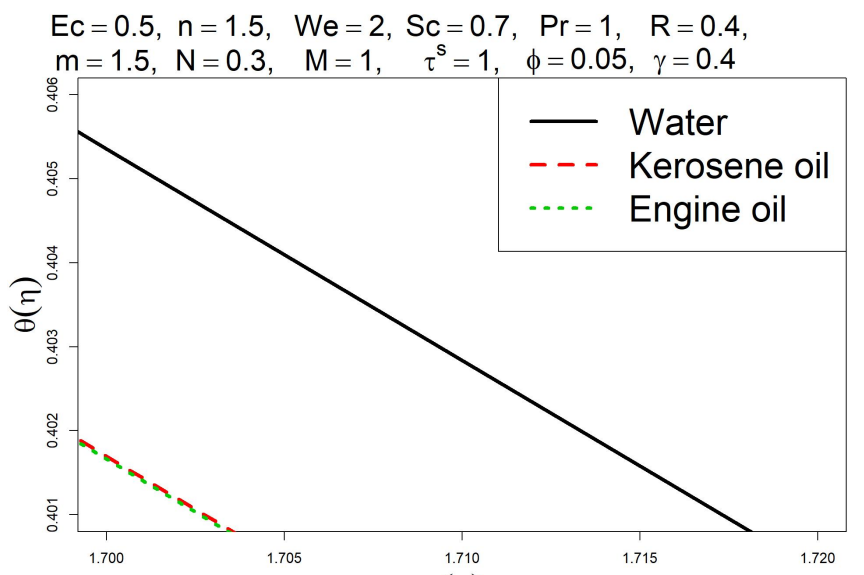

$(\eta)$

Fig. 15 Temperature profile for Shear thickening fluid with MWCNT 


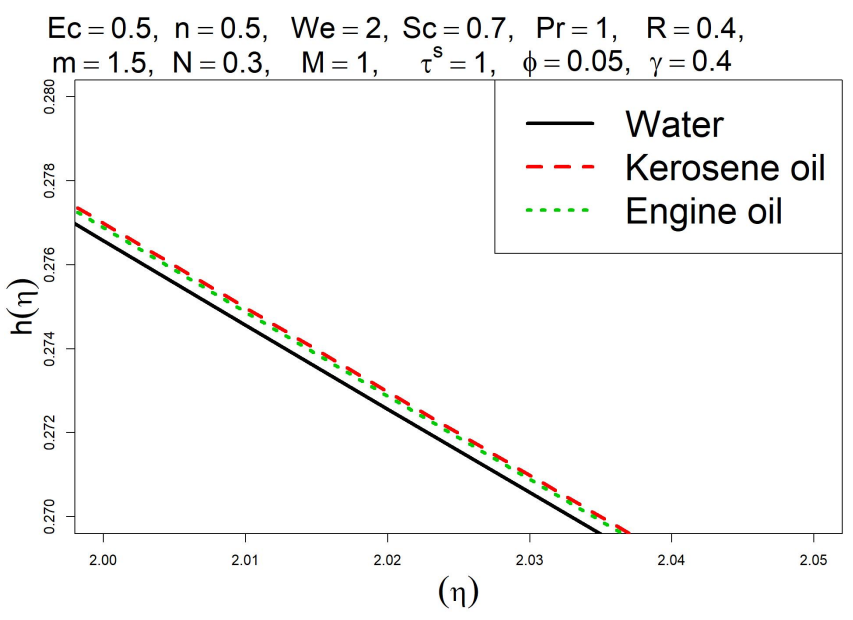

Fig. 16 Concentration profile for Shear thinning fluid with MWCNT

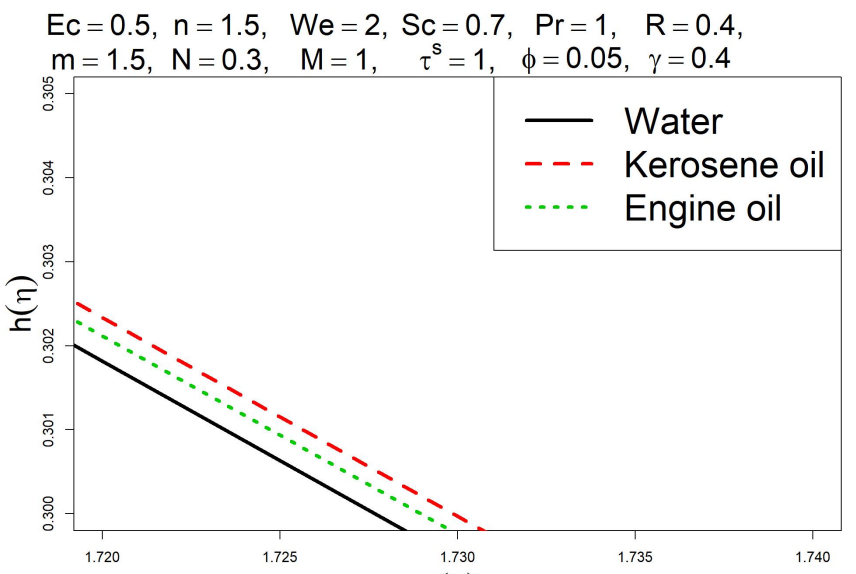

$(\eta)$

Fig. 17 Concentration profile for Shear thickening fluid with MWCNT

Table 1 A Comparison of the values of $f^{\prime \prime}(0)$ with $\mathrm{n}=1.0$, We $=0.0$, $\mathrm{M}=0.0, \gamma=0.0, \mathrm{Pr}=1.0, \mathrm{Ec}=0.0, \mathrm{Sc}=0.0, \mathrm{R}=0.0, \phi=0.0$ for different values of $\mathrm{m}$.

\begin{tabular}{|l|l|l|}
\hline$m$ & (Khan and Hashim, 2015) & Present study \\
\hline 0.0 & 0.6275549 & 0.6275627 \\
\hline 0.5 & 0.889544 & 0.8895524 \\
\hline 1.0 & 1.0 & 1.0 \\
\hline 1.5 & 1.061601 & 1.0616094 \\
\hline 3.0 & 1.148593 & 1.1486015 \\
\hline
\end{tabular}

Table 2 A Comparison of the values of $\theta^{\prime}(0)$ with $\mathrm{n}=1.0, \mathrm{We}=0.0, \mathrm{M}=0.0$, $\gamma=0.0, \operatorname{Pr}=1.0, \mathrm{Ec}=0.0, \mathrm{Sc}=0.0, \mathrm{R}=0.0, \phi=0.0$ for different values of $\mathrm{m}$.

\begin{tabular}{|l|l|l|}
\hline$m$ & (Khan and Hashim, 2015) & Present study \\
\hline 0.2 & 0.610202 & 0.6102159 \\
\hline 0.5 & 0.595201 & 0.5952237 \\
\hline 1.5 & 0.574730 & 0.5747717 \\
\hline 3.0 & 0.564662 & 0.5647180 \\
\hline 10.0 & 0.554951 & 0.5549518 \\
\hline
\end{tabular}

Table 3 Engineering parameters.

\begin{tabular}{|c|c|c|c|}
\hline M & \multirow{6}{*}{$\begin{array}{l}\mathrm{m}=1, \mathrm{we}=3.0 \\
\mathrm{Pr}=1.0, \mathrm{~N}=0.3, \\
\gamma=0.4, \mathrm{Ec}=0.5, \\
\mathrm{Sc}=0.7, \tau^{s}=0.3, \\
\tau^{s}=0.3, \\
\phi=0.05\end{array}$} & $\operatorname{Re}_{x}^{0.5} \mathrm{Cf}_{x}$ & $\operatorname{Re}_{x}^{0.5} \mathrm{Cf}_{x}$ \\
\hline & & $\mathrm{n}<1$ & $\mathrm{n}>1$ \\
\hline$\overline{0.0}$ & & -0.6972142 & -1.078309 \\
\hline 0.3 & & -0.7764511 & -1.249008 \\
\hline 0.6 & & -0.8461109 & -1.409071 \\
\hline 0.8 & & -0.8883215 & -1.510718 \\
\hline we & \multirow{6}{*}{$\begin{array}{l}\mathrm{m}=1, \mathrm{M}=3.0, \\
\operatorname{Pr}=1.0, \mathrm{~N}=0.3, \\
\gamma=0.4, \mathrm{Ec}=0.5, \\
\mathrm{Sc}=0.7, \tau^{s}=0.3, \\
\tau^{s}=0.3, \\
\phi=0.05\end{array}$} & $\operatorname{Re}_{x}^{0.5} \mathrm{Cf}_{x}$ & $\operatorname{Re}_{x}^{0.5} \mathrm{Cf}_{x}$ \\
\hline & & $\mathrm{n}<1$ & $n>1$ \\
\hline 2.0 & & -0.9520452 & -1.271478 \\
\hline 3.0 & & -0.8572339 & -1.354897 \\
\hline 3.5 & & -0.8192214 & -1.392846 \\
\hline 4.0 & & -0.7862717 & -1.428434 \\
\hline $\bar{M}$ & \multirow{6}{*}{$\begin{array}{l}\mathrm{m}=1, \mathrm{we}=3.0 \\
\operatorname{Pr}=1.0, \mathrm{~N}=0.3, \\
\gamma=0.4, \mathrm{Ec}=0.5, \\
\mathrm{Sc}=0.7, \tau^{s}=0.3, \\
\tau^{s}=0.3, \\
\phi=0.05\end{array}$} & $\mathrm{Re}_{x}^{-0.5} \mathrm{Nu}_{x}$ & $\operatorname{Re}_{x}^{-0.5} \mathrm{Nu}_{x}$ \\
\hline & & $\mathrm{n}<1$ & $\mathrm{n}>1$ \\
\hline 0.0 & & 0.3741720 & 0.4515941 \\
\hline 0.3 & & 0.3542758 & 0.4706764 \\
\hline 0.6 & & 0.3274603 & 0.4864306 \\
\hline 0.8 & & 0.3071349 & 0.4956823 \\
\hline we & \multirow{6}{*}{$\begin{array}{l}\mathrm{m}=1, \mathrm{M}=3.0, \\
\mathrm{Pr}=1.0, \mathrm{~N}=0.3, \\
\gamma=0.4, \mathrm{Ec}=0.5, \\
\mathrm{Sc}=0.7, \tau^{s}=0.3, \\
\tau^{s}=0.3, \\
\phi=0.05\end{array}$} & $\mathrm{Re}_{x}^{-0.5} \mathrm{Nu}_{x}$ & $\operatorname{Re}_{x}^{-0.5} \mathrm{Nu}_{x}$ \\
\hline & & $\mathrm{n}<1$ & $\mathrm{n}>1$ \\
\hline 2.0 & & 0.4060392 & 0.4836219 \\
\hline 3.0 & & 0.3760004 & 0.4990199 \\
\hline 3.5 & & 0.3630353 & 0.5056448 \\
\hline 4.0 & & 0.3513489 & 0.5116711 \\
\hline $\mathrm{M}$ & \multirow{6}{*}{$\begin{array}{l}\mathrm{m}=1, \mathrm{we}=3.0 \\
\operatorname{Pr}=1.0, \mathrm{~N}=0.3, \\
\gamma=0.4, \mathrm{Ec}=0.5, \\
\mathrm{Sc}=0.7, \tau^{s}=0.3, \\
\tau^{s}=0.3, \\
\phi=0.05\end{array}$} & $\mathrm{Re}_{x}^{-0.5} \mathrm{Sh}_{x}$ & $\operatorname{Re}_{x}^{-0.5} \mathrm{Sh}_{x}$ \\
\hline & & $\mathrm{n}<1$ & $\mathrm{n}>1$ \\
\hline 0.0 & & 0.5992370 & 0.6526943 \\
\hline 0.3 & & 0.5699964 & 0.6406981 \\
\hline 0.6 & & 0.5418290 & 0.6297297 \\
\hline 0.8 & & 0.5238310 & 0.6229667 \\
\hline we & \multirow{6}{*}{$\begin{array}{l}\mathrm{m}=1, \mathrm{M}=3.0, \\
\operatorname{Pr}=1.0, \mathrm{~N}=0.3, \\
\gamma=0.4, \mathrm{Ec}=0.5, \\
\mathrm{Sc}=0.7, \tau^{s}=0.3, \\
\tau^{s}=0.3, \\
\phi=0.05\end{array}$} & $\operatorname{Re}_{x}^{-0.5} \mathrm{Sh}_{x}$ & $\mathrm{Re}_{x}^{-0.5} \mathrm{Sh}_{x}$ \\
\hline & & $\mathrm{n}<1$ & $\mathrm{n}>1$ \\
\hline 2.0 & & 0.5897155 & 0.6324397 \\
\hline 3.0 & & 0.5750112 & 0.6416226 \\
\hline 3.5 & & 0.5688794 & 0.6455807 \\
\hline 4.0 & & 0.5634512 & 0.6491782 \\
\hline
\end{tabular}

\section{CONCLUSIONS}

MHD flow of Carreau nanofluid explored using CNT over a nonlinear stretched sheet with thermal radiation ,buoyancy and magnetic parameters has been studied. This investigation has explored that threshold value of momentum boundary layer is less than the threshold value of thermal and concentration boundary layers because of the effect of radiation and Local buoyancy parameter.

It was observed that the velocity profile diminished for $n<1$ and the contrary was true for $\mathrm{n}>1$ with increasing value of Weissenberg number (we). Moreover opposite behavior was observed for the temperature and concentration profiles. The temperature and thermal boundary layer thickness was decreased by the increasing Prandtl number (Pr). Increasing phenomenon of Schmidt number leads to low nanopartilce concentration.Investigators observed that radiation is one of the cause to reduce the boundary layer of Concentration with Local buoyancy paramter relative to thermophoretic parameter. Investigators noticed that due to thermal conductivity and specific heat of Water, water based carbon nanotube(SWCNT and MWCNT) profiles are enhanced when compared to the remaning base fluids except at concentration profile, because of thermophoretic parameter.

This study provides information and limitations of the use of the Single walled carbon nanotube with water as base fluid. The thermal performance of the nanofluids are not much different and it may be important to observe other nanofluids which may have higher heat transfer rate. $\mathrm{Nu}$ - 
merical Simulations were obtained by bvp solver using R-Software.

\section{ACKNOWLEDGEMENTS}

Investigators are extremely thankful to Koneru Lakshmaiah Education Foundation and Jawaharlal Nehru Architecture and Fine Arts University for giving a lot of support during this research work at the Department of Mathematics, Koneru Lakshmaiah Education Foundation,Guntur and Department of Digital Technology, Jawaharlal Nehru Architecture and Fine Arts University,Hyderabad.

\section{NOMENCLATURE}

$x, y \quad$ Cartesian coordinates

$p, \mathrm{q} \quad$ Velocity components in $x, y$ directions respectively

$B \quad$ Magnetic field intensity

$B_{0} \quad$ Constant magnetic field intensity

$g \quad$ Gravitational acceleration

$T \quad$ Temperature of the flow field

$C \quad$ Species concentration

$T_{w} \quad$ Temperature at the wall

$T_{\infty} \quad$ Temperature of the fluid outside the boundary layer

$T_{\text {ref }} \quad$ Reference temperature

$C_{w} \quad$ Concentration at the wall

$C_{\infty} \quad$ Concentration outside the boundary layer

$D \quad$ Diffusion coefficient

$V_{T} \quad$ Thermophoretic velocity

$k_{1} \quad$ Thermophoretic coefficient

$c_{p} \quad$ Specific heat

$k_{n f} \quad$ Thermal conductivity of nanofluid

$k_{b f} \quad$ Thermal conductivity of basefluid

$k_{C N T} \quad$ Thermal conductivity of CNT

$k^{s} \quad$ Mean absorption coefficient

$w e_{x} \quad$ LocalWeissenberg number

$G r_{x} \quad$ Local Grashof number

$R e_{x} \quad$ Local Reynolds number

$\mathrm{Pr} \quad$ Prandtl number

Ec Eckert number

Sc Schmidt number

$M \quad$ Magnetic parameter

$N \quad$ Concentration buoyancy parameter

$R \quad$ Radiation parameter

$n \quad$ Power law index

$m, \mathrm{~b} \quad$ positive real numbers relating to the stretching

Greek Symbols

$\rho \quad$ Fluid density

$\gamma \quad$ Local buoyancy parameter

$\tau \quad$ Material time constant

$\beta_{T} \quad$ Thermal expansion coefficients of temperature

$\beta_{C} \quad$ Thermal expansion coefficients of Concentration

$\sigma^{s} \quad$ Stefan-Boltzmann constant

$\nu_{n f} \quad$ Coefficient of kinematics viscosity of nanofluid

$\mu_{n f} \quad$ Coefficient of viscosity of nanofluid

$\mu_{b f} \quad$ Coefficient of viscosity of basefluid

$\phi \quad$ Nanoparticle volume fraction

$\sigma_{n f} \quad$ Electrical conductivity of nanofluid

$\sigma_{b f} \quad$ Electrical conductivity of basefluid

$\sigma_{C N T} \quad$ Electrical conductivity of CNT

$\psi \quad$ Stream function

$\eta \quad$ Similarity variable

$\theta \quad$ Dimensionless fluid temperature

$h \quad$ Dimensionless fluid Concentration

$\tau^{s} \quad$ Thermophoretic parameter

\section{REFERENCES}

Ahmad, K., and Nazar, R., 2011, "Magnetohydrodynamic three dimensional flow and heat transfer over a stretching surface in a viscoelastic fluid." J Sci Technol, 3, 1-14.

https://publisher.uthm.edu.my/ojs/index.php/JST/article/view/195 .

Andersson, H., 1992, "MHD flow of a viscoelastic fluid past a stretching surface." Acta Mech, 95, 227-230.

https://doi.org/10.1007/BF01170814.

Anwar, M., and Rasheed, A., 2017, "A microscopic study of MHD fractional inertial flow through Forchheimer medium." Chin J Phys, 55, 1690-703.

https://doi.org/10.1016/j.cjph.2017.05.011.

Carreau, P., 1972, "Rheological equations from molecular network theories," Trans Soc Rheol 1972, 16, 99-127.

https://doi.org/10.1122/1.549276.

Chamkha, A., 1997, "Hydromagnetic natural convection from an isothermal inclined surface adjacent to a thermally stratified porous medium." Int J Eng Sci, 35, 975-86. https://doi.org/10.1016/S0020-7225(96)00122-X.

Haq, R., Noor, N., and Khan, 2016, "Numerical simulation of water based magnetite nanoparticles between two parallel disks." Adv Powder Technol, 27, 1568-75.

https://doi.org/10.1016/j.apt.2016.05.020.

Hayat, T., Rafique, K., Muhammad, T., Alsaedi, A., and Ayub, M., 2018, "Carbon nanotubes significance in Darcy-Forchheimer flow," Results Phys 2018, 8, 26-33.

https://doi.org/10.1016/j.rinp.2017.11.022.

Khan, M., and Hashim, 2015, "Boundary layer flow and heat transfer to Carreau fluid over a nonlinear stretching sheet." AIP Advances, 5, 107203.

https://doi.org/10.1063/1.4932627.

Khan, M., Irfan, M., and ., W.K., 2017, "Impact of nonlinear thermal radiation and gyrotactic microorganisms on the Magneto-Burgers nanofluid." Int J Mech Sci, 130, 375-82.

https://doi.org/10.1016/j.ijmecsci.2017.06.030.

Lu, D., Li, Z., Ramzan, M., Shafee, M., and Chung, J., 2019, "Unsteady squeezing carbon nanotubes based nano-liquid flow with Cattaneo-Christov heat flux and homogeneous-heterogeneous reactions." Sci Rep 2018, 9, 169-178.

https://doi.org/10.1007/s13204-018-0899-1.

Lu, D., Ramzan, M., Ahmad, S., Chung, J., and Farooq, U., 2018, “A numerical treatment of MHD radiative flow of Micropolar nanofluid with homogeneous-heterogeneous reactions past a nonlinear stretched surface." Sci Rep 2018, 8, 12431. https://doi.org/10.1038/s41598-018-30965-x.

Pavlov, K., 1974, "Magnetohydrodynamic flow of an incompressible viscous fluid caused by the deformation of a plane surface." Magnetohydrodynamic, 10, 146-148. http://doi.org/10.22364/mhd.

Takhar, H., Chamkha, A., and Nath., G., 1999, "Unsteady flow and heat transfer on a semi-infinite flat plate with an aligned magnetic field," Int $J$ Eng Sci 1999l, 37, 1723-36.

https://doi.org/10.1016/S0020-7225(98)00144-X. 
Takhar, H., Chamkha, A., and Nath, G., 2001, "Unsteady threedimensional MHD-boundary-layer flow due to the impulsive motion of a stretching surface." Acta Mechl, 146, 59-71.

https://doi.org/10.1007/BF01178795.
Vasu, B., and Ray, A., 2019, "Numerical study of Carreau nanofluid flow past vertical plate with the Cattaneo-Christov heat flux model." IntJNumerMethods Heat Fluid Flow 2019, 29, 702-723.

https://doi.org/10.1108/HFF-03-2018-0104. 